\title{
Tree-level interference in vector boson fusion production of $\mathrm{Vh}$
}

\author{
Daniel Stolarski॰ $\odot^{*}$ and Yongcheng $\mathrm{Wu} \odot^{\dagger}$ \\ Ottawa-Carleton Institute for Physics, Carleton University, 1125 Colonel By Drive, \\ Ottawa, Ontario K1S 5B6, Canada
}

(Received 25 June 2020; accepted 13 August 2020; published 31 August 2020)

\begin{abstract}
Vector boson scattering is a well known probe of electroweak symmetry breaking. Here we study a related process of two electroweak vector bosons scattering into a vector boson and a Higgs boson $(V V \rightarrow V h, V=W, Z)$. This process exhibits tree level interference and grows with energy if the Higgs couplings to electroweak bosons deviate from their Standard Model values. Therefore, this process is particularly sensitive to the relative sign of the ratio of the coupling between the Higgs and the $W$ and $Z$, $\lambda_{W Z}$. In this work we show that a high energy lepton collider is well suited to study this process through vector boson fusion, estimate the potential sensitivity to this ratio, and show that a relatively modest amount of data can exclude $\lambda_{W Z} \simeq-1$.
\end{abstract}

DOI: $10.1103 /$ PhysRevD.102.033006

\section{INTRODUCTION}

In the Standard Model (SM), the Higgs boson is a necessary ingredient to keep longitudinal gauge boson scattering unitary at high energy [1-6]. Even in the presence of the Higgs, if its couplings deviate from those predicted by the SM, longitudinal gauge boson scattering will still grow with energy and new physics is needed to make the theory unitary [7-9]. Therefore, the experimental discovery of the Higgs [10,11] is insufficient to probe the nature of the unitarization of longitudinal gauge boson scattering; detailed measurements of its couplings are necessary.

Vector boson scattering $(V V \rightarrow V V, V=Z, W)$ is an extremely well studied subject (for a review see [12]). One can also study a similar process $V V \rightarrow h h$ [9], where $h$ is the Higgs boson. More complicated processes involving gauge bosons can also be used to measure various Higgs couplings even when the Higgs is not one of the external particles [13]. In this work, we study another class of process:

$$
V V \rightarrow V h, \quad V=W, Z
$$

Like the two processes above, this one also exhibits growth with energy if the couplings of the Higgs to massive gauge

\footnotetext{
*stolar@physics.carleton.ca

ycwu@physics.carleton.ca
}

Published by the American Physical Society under the terms of the Creative Commons Attribution 4.0 International license. Further distribution of this work must maintain attribution to the author(s) and the published article's title, journal citation, and DOI. Funded by SCOAP ${ }^{3}$. bosons deviate from the Standard Model prediction. This process is especially sensitive to the ratio of the coupling of the Higgs to the $W$ relative to that of the $Z$. In particular, if we define $\kappa_{W}\left(\kappa_{Z}\right)$ as the deviation of the $W(Z)$ coupling to the Higgs from the SM prediction $\left(\kappa_{W}=\kappa_{Z}=1\right.$ in the SM, see Eq. (5) below for more details), then we can define:

$$
\lambda_{W Z}=\frac{\kappa_{W}}{\kappa_{Z}},
$$

as the specified ratio. The process in Eq. (1) exhibits treelevel interference effects between $W$ and $Z$ mediated processes, and the matrix element has a term that grows with energy proportional to $\lambda_{W Z}-1$.

Because a heavy gauge boson collider is not feasible, the typical gauge boson scattering is studied experimentally via vector boson fusion (VBF), where $W$ or $Z$ 's are radiated off the initial state fermions and then scatter off one another. Analytic understanding can be further gained via the effective $W$ approximation [14-16], by treating the radiated gauge bosons as approximately on-shell. Gauge boson scattering has been observed at the LHC [17-20], but a lepton collider is a cleaner environment which may allow for more precise measurements. A lepton collider is a particularly good machine for precision studies of the Higgs, and planning has begun for several different machines including the ILC $[21,22]$, circular colliders CEPC [23] and FCC-ee [24,25], and CLIC [26,27] which has higher center of mass (CM) energies, with designs ranging from 1.5 to $3 \mathrm{TeV}$.

\footnotetext{
${ }^{1}$ As this work was being completed, a study of this and other process at a high energy muon collider was posted in [28].
} 
TABLE I. High energy behavior of the polarized process given in Eq. (4) as a function of Mandelstam $s$ for the matrix element $\mathcal{M}$ and the differential cross section $d \sigma$. The second and forth columns are for the $s$ - and $t$-channel processes individually, while the third and fifth columns are for the $s$ - and $t$-channel processes summed. The different rows are for different combinations of polarizations of gauge bosons whether they are transverse (T) or longitudinal (L).

\begin{tabular}{lcccc}
\hline \hline & $\mathcal{M}_{s / t}$ & $\mathcal{M}_{s}+\mathcal{M}_{t}$ & $d \sigma_{s / t}$ & $d \sigma_{\text {tot }}$ \\
\hline TTT & $\frac{1}{\sqrt{s}}$ & $\frac{1}{\sqrt{s}}$ & $\frac{1}{s^{2}}$ & $\frac{1}{s^{2}}$ \\
LTT & $s^{0}$ & $s^{0}$ & $\frac{1}{s}$ & $\frac{1}{s}$ \\
LLT & $\sqrt{s}$ & $\frac{1}{\sqrt{s}}$ & $s^{0}$ & $\frac{1}{s^{2}}$ \\
LLL & $s$ & $s^{0}$ & $s$ & $\frac{1}{s}$ \\
\hline \hline
\end{tabular}

Therefore, in this work we study the process of Eq. (1) with VBF at a high energy lepton collider:

$$
\begin{aligned}
& e^{+} e^{-} \rightarrow \nu_{e} \bar{\nu}_{e} Z h, \\
& e^{+} e^{-} \rightarrow \nu_{e} e W h .
\end{aligned}
$$

This process grows with the center of mass energy of the lepton collider, with CLIC being a particularly good machine for its study. As we will show, this process is very sensitive to modification of $\lambda_{W Z}$ from the SM prediction because of the tree-level interference. The work here is complimentary to others that use interference effects to measure $\lambda_{W Z}$ such as $e^{+} e^{-} \rightarrow W^{+} W^{-} h$ [29] or interference of tree and loop effects in $h \rightarrow 4 \ell$ [30].

One particularly interesting scenario is that when $\lambda_{W Z}$ is negative relative to the SM prediction. Tree-level processes without interference effects such as decays of $h \rightarrow Z Z^{*}$ $[31,32]$ and $h \rightarrow W W^{*}[33,34]$ are only sensitive to $\left|\lambda_{W Z}\right|$. Fits to the couplings by the experimental collaborations [3537] measure $\lambda_{W Z}$ with approximately $10 \%$ precision but have almost no discriminating power between positive and negative values of $\lambda_{W Z}{ }^{2}$ The ultimate LHC sensitivity on this ratio is projected to be about $2 \%$ [38], but as far as we are aware, there has been no study on the sensitivity to the sign from rate measurements at the LHC. Negative values of $\lambda_{W Z}$ can arise in models with scalars that have higher isospin representations [39] such as the Georgi-Machacek [40] model. In that case, however, the processes of Eq. (3) will be many times larger than the SM prediction and can be easily measured with a high energy lepton collider.

The remainder of this paper is structured as follows: in Sec. II we explore the behavior of the $2 \rightarrow 2$ process focusing on the growth with energy, and in Sec. III we describe the process in vector boson fusion at a lepton

\footnotetext{
${ }^{2}$ The $13 \mathrm{TeV}$ CMS analysis [36] actually has a best fit value that is negative, and the $13 \mathrm{TeV}$ ATLAS analysis [37] does not consider negative values of $\lambda_{W Z}$.
}

collider and show the importance of tree-level interference. In Sec. IV we conduct a phenomenological study including backgrounds and show how the analysis can be improved by taking differential distributions into account, and finally a summary is given in Sec. V.

\section{II. $2 \rightarrow 2$ PROCESSES}

We begin by analyzing the $2 \rightarrow 2$ processes

$$
\begin{aligned}
W^{+} W^{-} & \rightarrow Z h, \\
W^{ \pm} Z & \rightarrow W^{ \pm} h,
\end{aligned}
$$

to understand the large interference effects that can occur. We parametrize the coupling of the Higgs $(h)$ to the $W$ and $Z$ as

$$
\mathcal{L}=h\left(\kappa_{W} g m_{W} W_{\mu}^{+} W_{\mu}^{-}+\kappa_{Z} g \frac{m_{Z}^{2}}{2 m_{W}} Z_{\mu} Z_{\mu}\right),
$$

where $g$ is the SM $S U(2)$ gauge coupling, and in the Standard Model $\kappa_{W}=\kappa_{Z}=1$, with values away from one parametrizing deviation from the SM prediction.

Both processes in Eq. (4) have contributions from $s$ and $t$-channel diagrams (the Feynman diagrams can be read from the VBF Feynman diagrams below in Fig. 2). In the first (second) process, the $s$-channel diagram is proportional to $\kappa_{Z}\left(\kappa_{W}\right)$, while the $t$-channel diagrams are proportional to $\kappa_{W}\left(\kappa_{Z}\right)$. We can divide the process into subprocesses based on the number of transversely vs longitudinally polarized gauge bosons in this process. Direct calculation shows that the matrix elements with two or three longitudinal gauge bosons grow with energy. This is analogous to the classic studies of $W W \rightarrow W W$ scattering [1-6], and even though the $s$ and $t$-channel processes individually grow with energy, their sum displays a cancellation such that the final amplitude does not grow with energy and the theory remains unitary at high energy. We summarize the high energy behavior of the polarized matrix elements in Table I for different combinations of vector polarizations.

We can analyze the case $W_{L} W_{L} \rightarrow Z_{L} h$ in more detail. We expand the matrix elements for the $s$ - and $t$-channel processes in the high energy limit:

$$
\begin{gathered}
\mathcal{M}_{s}\left(W_{L}^{+} W_{L}^{-} \rightarrow Z_{L} h\right) \\
=\frac{\kappa_{Z} g^{2} \cos \theta}{4 m_{W}^{2}}\left(s-m_{h}^{2}+2 m_{Z}^{2}\right)+\mathcal{O}\left(\frac{1}{s}\right), \\
\mathcal{M}_{t}\left(W_{L}^{+} W_{L}^{-} \rightarrow Z_{L} h\right) \\
=\frac{\kappa_{W} g^{2}}{4 m_{W}^{2}}\left(\cos \theta\left(-s+2 m_{W}^{2}+m_{Z}^{2}-m_{h}^{2}\right)+8 m_{W}^{2} \frac{\cos \theta}{\sin ^{2} \theta}\right) \\
+\mathcal{O}\left(\frac{1}{s}\right),
\end{gathered}
$$



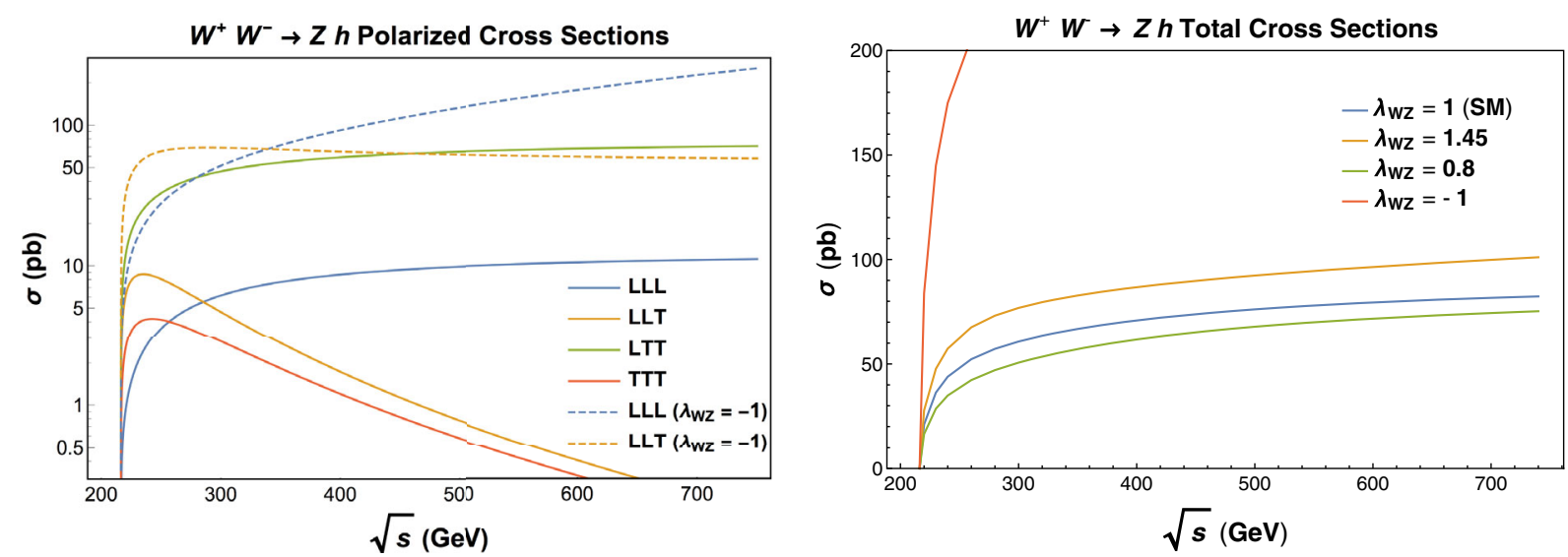

FIG. 1. Left: polarized cross sections of the process $W^{+} W^{-} \rightarrow Z h$ as a function of center of mass energy $\sqrt{s}$ categorized by the number of transversely (T) or longitudinally (L) polarized vectors in the process. The solid lines are SM results while the dashed lines have $\lambda_{W Z}=-1$. Right: total cross sections for the same process. The blue line is the SM result, while other lines have different values of $\lambda_{W Z}$. We have taken $\kappa_{W}=1$ in all cases. Note that the left plot is on a log-scale, while the right one is linear.

where $\theta$ is the scattering angle in the center of momentum frame. The singularity in the forward $(\theta=0)$ and backward $(\theta=\pi)$ limits in the $t$-channel diagrams are artifacts of the high energy expansion and are cut off by masses in the full expression. Adding the two matrix elements:

$\mathcal{M}\left(W_{L}^{+} W_{L}^{-} \rightarrow Z_{L} h\right)=\frac{g^{2} \kappa_{Z} \cos \theta}{4 m_{W}^{2}}\left(1-\lambda_{W Z}\right) s+\mathcal{O}\left(s^{0}\right)$,

where $\lambda_{W Z}$ is defined in Eq. (2) and equal to one in the SM. We see that at high energy, the SM predicts that the matrix element of this process goes to a constant, and thus the cross section falls, preserving unitarity. On the other hand, if there is new physics that modifies the ratio of the coupling of the Higgs to the electroweak gauge bosons, $\lambda_{W Z} \neq 1$, then this amplitude and thus the cross section will grow quadratically with center of mass energy. This growth will eventually be cut off by new resonances or other effects of new physics. A particularly interesting case is that of $\lambda_{W Z} \simeq-1$. This is impossible to distinguish from the Standard Model prediction without an interference measurement, and the process studied here is extremely sensitive to this scenario.

We now look at a full calculation of the cross section in Fig. 1. On the left panel, looking first at the solid lines which are the SM prediction, the cross section is dominated by the process with two transverse and one longitudinal gauge boson (LTT). ${ }^{3}$ Of these, the largest are the processes where one of the initial states is longitudinal, and the two

\footnotetext{
${ }^{3}$ Note that, the energy dependence of the cross section in LTT and LLL configurations does not behave as expected shown in Table I. This is mainly due to the fact that in the forward and backward region $(\cos \theta= \pm 1)$, the high energy expansion is different. When integrating over $\cos \theta$, we will obtain different dependence on the scattering energy. However, the overall cancellation between $s$ and $t / u$ contributions is not ruined.
}

transversely polarized gauge bosons have opposite chirality. If we now turn to the dashed lines which have $\lambda_{W Z}=-1$, we see the dramatic growth with energy of the process with all longitudinal gauge bosons, confirming the analysis of Eq. (8). We can also see that the process with two longitudinal gauge bosons is significantly enhanced.

On the right panel of Fig. 1 we study how the total cross section as a function of energy varies with $\lambda_{W Z}$, and we can again see that for $\lambda_{W Z} \simeq-1$, the cross section is much larger than the SM prediction for all energies. Even for moderate modifications of $\lambda_{W Z}$ there can be significant changes to the cross section. Analyzing the isospin related processes $W^{ \pm} Z \rightarrow W^{ \pm} h$ gives analogous results. Therefore we see that because of the two processes that grow with energy, and the cancellation that occurs only at the SM value of $\lambda_{W Z}$, measurement of this process is a very sensitive probe of this coupling ratio. Unfortunately, an electroweak gauge boson collider is not feasible, so directly measuring these processes is not possible. In what follows we turn to the more realistic case of measuring vector boson scattering as a subprocess in a lepton collider.

\section{VH PRODUCTION THROUGH VBF}

The processes we are considering is the $V+$ Higgs $(V=W, Z)$ production through VBF at a lepton collider ${ }^{4}$ :

$$
\begin{aligned}
& e^{+} e^{-} \rightarrow \nu_{e} \bar{\nu}_{e} Z h, \\
& e^{+} e^{-} \rightarrow \nu_{e} e W h .
\end{aligned}
$$

The main Feynman diagrams for these two processes are shown in Fig. 2. Figure 2(a), 2(b), 2(d), and 2(f) contain the

\footnotetext{
${ }^{4}$ We use electrons for the initial state, but the analysis is very similar at a muon collider.
} 


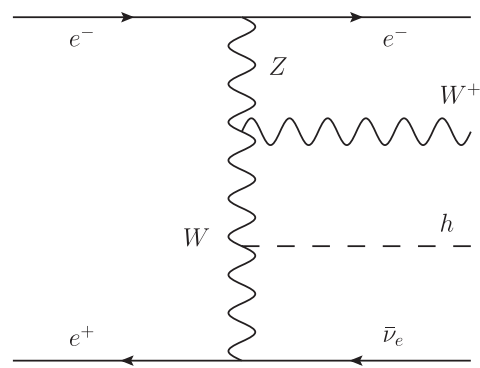

(a)

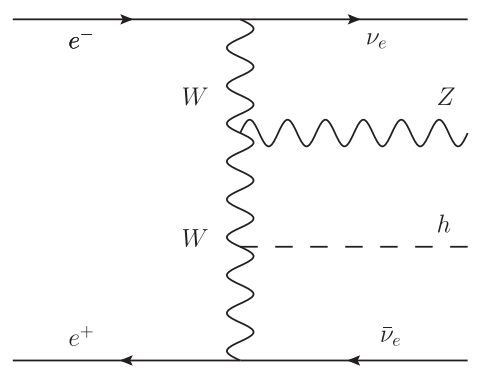

(d)

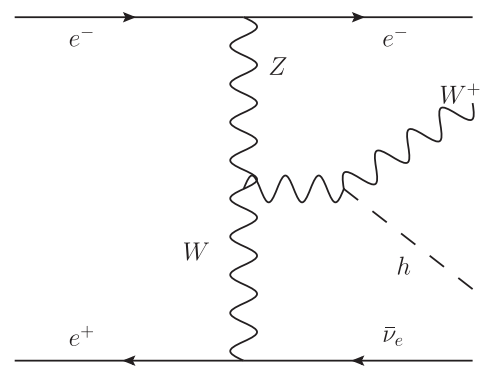

(b)

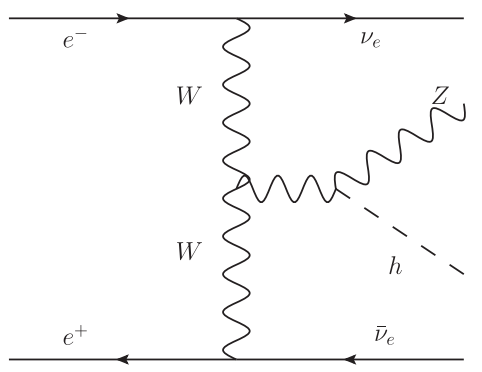

(e)

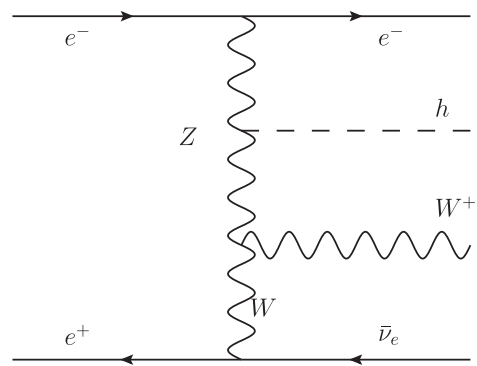

(c)

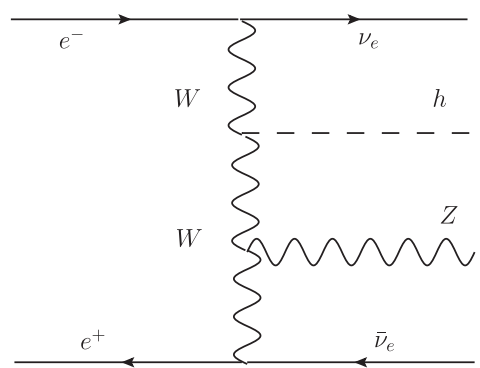

(f)

FIG. 2. The Feynman diagrams for $W h(\mathrm{a}-\mathrm{c})$ and $Z h(\mathrm{~d}-\mathrm{f})$ production through VBF processes at a lepton collider.

$h W W$ vertex, while Fig. 2(c) and 2(e) rely on the $h Z Z$ vertex. The cross section as a function of collision energy for three different polarizations are also shown in Fig. 3. (For the cross section calculation, we require $p_{T}^{\ell}>5 \mathrm{GeV}$ and $\left|\eta_{\ell}\right|<3.5$.) The cross section grows as the collision energy increases. To fully utilize the potential of these two processes, we will consider both the $3 \mathrm{TeV}$ and $1.5 \mathrm{TeV}$ scenarios at CLIC [27]. We also note that polarization of the electron beam can significantly increase the cross section in the left-handed configuration.

We denote the contribution of the matrix element square from $h W W$ couplings as $\left|\mathcal{M}_{W}\right|^{2}$ (e.g., from (a,b) or (d,f) only), the contribution from $h Z Z$ couplings as $\left|\mathcal{M}_{Z}\right|^{2}$ (from (c) or (e) only) and the interference term as $\mathcal{M}_{W Z}^{2}$. Then the total matrix element square for either $W h$ or $Z h$ processes can be written as (with the dependence on the $\kappa$ of relevant couplings):

$$
|\mathcal{M}|^{2}=\kappa_{W}^{2}\left|\mathcal{M}_{W}\right|^{2}+\kappa_{W} \kappa_{Z} \mathcal{M}_{W Z}^{2}+\kappa_{Z}^{2}\left|\mathcal{M}_{Z}\right|^{2} .
$$

The total cross section is

$$
\sigma=\kappa_{W}^{2} \sigma_{W}+\kappa_{W} \kappa_{Z} \sigma_{W Z}+\kappa_{Z}^{2} \sigma_{Z}
$$

Following the polarization configuration presented in [27], the individual contributions are listed in Table II for three different $\sqrt{s}$. We find that, besides the case $\sqrt{s}=350 \mathrm{GeV}$ where the production rate is small, the interference effect is
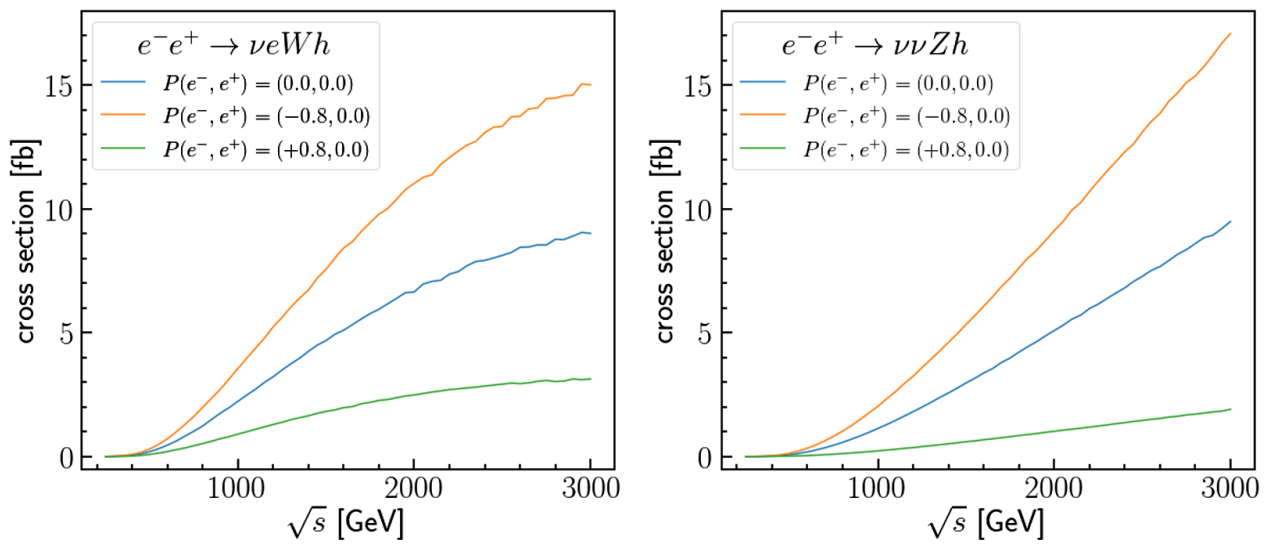

FIG. 3. The cross section of two processes as a function of $\sqrt{s}$ for there different polarizations. The cross sections are calculated using MadGraph5_aMC@NLO [41] with $p_{T}^{\ell}>10 \mathrm{GeV}$ and $\left|\eta^{\ell}\right|<3.5$. 
TABLE II. The individual contributions to total cross section for $W h$ and $Z h$ VBF processes at different collision energies and different polarizations. The cross section is obtained from MadGraph5_aMC@NLO with cuts: $p_{T}^{\ell}>10 \mathrm{GeV}$ and $\left|\eta_{\ell}\right|<3.5$. The polarization configuration is following those in [27].

\begin{tabular}{|c|c|c|c|c|c|}
\hline \multicolumn{2}{|c|}{$\sigma[\mathrm{fb}]$} & \multicolumn{2}{|c|}{$W h$} & \multicolumn{2}{|c|}{$Z h$} \\
\hline$\sqrt{s}[\mathrm{GeV}]$ & & $P\left(e^{-}\right)=-80 \%$ & $P\left(e^{-}\right)=80 \%$ & $P\left(e^{-}\right)=-80 \%$ & $P\left(e^{-}\right)=80 \%$ \\
\hline 350 & $\begin{array}{c}\sigma_{Z} \\
\sigma_{W} \\
\sigma_{W Z}\end{array}$ & $\begin{array}{r}6.81 \times 10^{-3} \\
3.85 \times 10^{-2} \\
-3.94 \times 10^{-3}\end{array}$ & $\begin{array}{r}2.46 \times 10^{-3} \\
8.27 \times 10^{-2} \\
-2.22 \times 10^{-3}\end{array}$ & $\begin{array}{r}1.08 \times 10^{-2} \\
1.49 \times 10^{-2} \\
-1.03 \times 10^{-2}\end{array}$ & $\begin{array}{r}2.91 \times 10^{-3} \\
1.65 \times 10^{-3} \\
-1.16 \times 10^{-3}\end{array}$ \\
\hline 1500 & $\begin{array}{c}\sigma_{Z} \\
\sigma_{W} \\
\sigma_{W Z}\end{array}$ & $\begin{array}{r}8.25 \times 10^{0} \\
1.22 \times 10^{1} \\
-1.28 \times 10^{1}\end{array}$ & $\begin{array}{r}3.18 \times 10^{0} \\
4.11 \times 10^{0} \\
-5.46 \times 10^{0}\end{array}$ & $\begin{array}{r}3.85 \times 10^{0} \\
6.85 \times 10^{0} \\
-5.38 \times 10^{0}\end{array}$ & $\begin{array}{l}4.25 \times 10^{-1} \\
7.66 \times 10^{-1} \\
5.93 \times 10^{-1}\end{array}$ \\
\hline 3000 & $\begin{array}{c}\sigma_{Z} \\
\sigma_{W} \\
\sigma_{W Z}\end{array}$ & $\begin{array}{r}3.51 \times 10^{1} \\
4.31 \times 10^{1} \\
-6.32 \times 10^{1}\end{array}$ & $\begin{array}{r}1.34 \times 10^{1} \\
1.50 \times 10^{1} \\
-2.52 \times 10^{1}\end{array}$ & $\begin{array}{r}1.87 \times 10^{1} \\
2.97 \times 10^{1} \\
-3.13 \times 10^{1} \\
\end{array}$ & $\begin{array}{r}2.09 \times 10^{0} \\
3.27 \times 10^{0} \\
-3.45 \times 10^{0} \\
\end{array}$ \\
\hline
\end{tabular}

very large, comparable or even larger than the individual contributions of $\sigma_{W}$ and $\sigma_{Z}$. This significant interference effect offers the opportunity to measure the $\kappa_{W}, \kappa_{Z}$ as well as $\lambda_{W Z}$ precisely. It also demonstrates the dramatic sensitivity to the sign of $\lambda_{W Z}$. In the following section, we conduct a detailed phenomenological study of this channel including backgrounds and realistic experimental cuts.

\section{PHENOMENOLOGY STUDY}

A measurement of the cross section of the processes studied here can be translated into a measurement of $\lambda_{W Z}$. This process is particularly sensitive to the sign of this parameter because of the tree-level interference, and can thus relatively easily rule out the case with $\lambda_{W Z}<0$ where the destructive interference effect in the SM turns into a constructive effect. Here we perform a realistic study of this cross section measurement.

\section{A. Total rate measurement}

The signal processes we consider are

$$
\begin{aligned}
& e^{-} e^{+} \rightarrow e^{ \pm} \nu_{e} W^{\mp} h, \\
& e^{-} e^{+} \rightarrow \nu_{e} \bar{\nu}_{e} Z h .
\end{aligned}
$$

We consider the final state containing two isolated leptons, two $b$-jets (from Higgs decay) and $\mathbb{E}_{T}$. Thus, the dominant backgrounds ${ }^{5}$ come from

$$
e^{-} e^{+} \rightarrow t \bar{t} \rightarrow b \bar{b} \ell^{-} \ell^{+} \nu_{\ell} \bar{\nu}_{\ell}
$$

\footnotetext{
${ }^{5}$ Another process with the same final states is associated production of a Higgs and two vector bosons, $e^{-} e^{+} \rightarrow V V h$. This process also exhibits tree-level interference so it can be thought of as signal rather than background. The cross section is small and the topology is quite different from the signal, so it has a negligible contribution to this analysis after the cuts.
}

$$
\begin{aligned}
& e^{-} e^{+} \rightarrow e^{ \pm} \nu_{e} W^{ \pm} Z \rightarrow e^{ \pm} \nu_{e} \ell^{\mp} \nu_{\ell} b \bar{b}, \\
& e^{-} e^{+} \rightarrow \nu_{e} \bar{\nu}_{e} Z Z \rightarrow \nu_{e} \bar{\nu}_{e} \ell^{-} \ell^{+} b \bar{b}, \\
& e^{-} e^{+} \rightarrow Z h, \quad Z \rightarrow \ell^{-} \ell^{+}, \quad h \rightarrow b \bar{b},
\end{aligned}
$$$$
e^{-} e^{+} \rightarrow Z W^{+} W^{-}, \quad Z \rightarrow b \bar{b}, \quad W^{+} \rightarrow \ell^{+} \nu_{\ell},
$$$$
W^{-} \rightarrow \ell^{-} \bar{\nu}_{\ell}
$$

$e^{-} e^{+} \rightarrow Z Z Z, \quad Z \rightarrow b \bar{b}, \quad Z \rightarrow \ell^{-} \ell^{+}, \quad Z \rightarrow \nu_{\ell} \bar{\nu}_{\ell}$.

The events are generated using MadGraph5_aMC@NLO [41] with PYTHIA8 [42] used for showering and hadronization. The detector effects are simulated with DELPHES [43] using the CLIC card [44]. In order to improve the sensitivity, we simulate both $3 \mathrm{TeV}$ and $1.5 \mathrm{TeV}$ events with $P\left(e^{-}\right)=-0.8$ for the electron beam which are two scenarios for CLIC with 4000 and $2000 \mathrm{fb}^{-1}$ luminosity respectively [27].

The analysis is separated into two channels aiming on $W h$ and $Z h$ production respectively. Based on the distributions and the event topology of the signal and background shown in Fig. 4, the following cuts are applied on the events which are also listed in Table III:

(1) $p_{T}^{\ell}>20 \mathrm{GeV}$.

(2) $p_{T}^{j}>20 \mathrm{GeV}$.

(3) Two isolated leptons:

(a) For Wh channel, at least one electron which directly comes from the beam remnants.

(b) For $Z h$ channel, one pair of opposite-sign sameflavor (OSSF) leptons is required.

(4) Two jets tagged as $b$-jet.

(5) The invariant mass of the two $b$-jet: $95 \mathrm{GeV} \leq$ $m_{b b} \leq 130 \mathrm{GeV}$.

(6) The invariant mass of the two isolated leptons: 

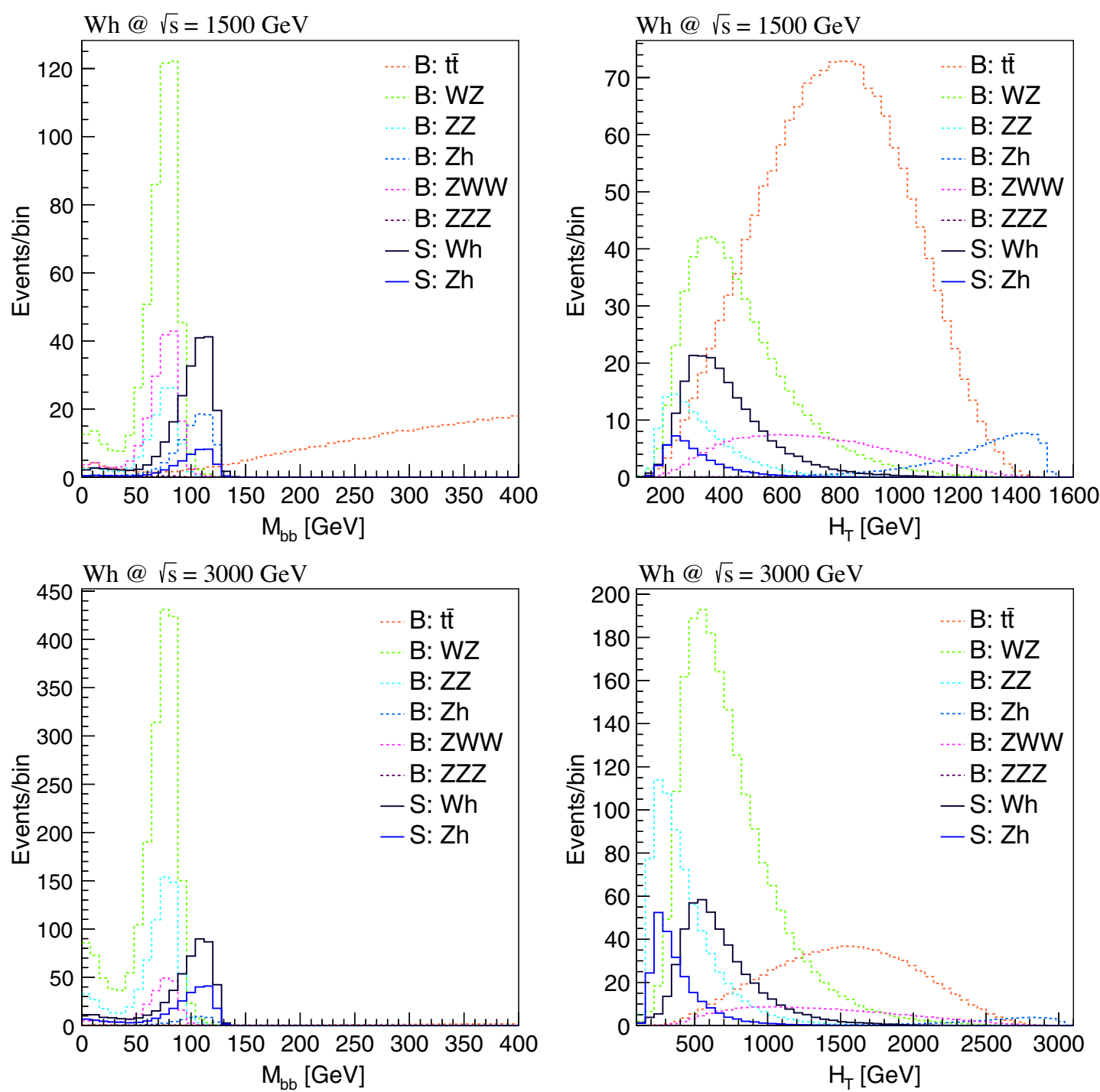

FIG. 4. The distribution of $m_{b b}$ and $H_{T}$ in $W h$ channel at $\sqrt{s}=1500 \mathrm{GeV}$ (Upper panels) and $\sqrt{s}=3000 \mathrm{GeV}$ (Lower panels) with only the basic cuts listed in Table III applied. Note that for $\sqrt{s}=3000 \mathrm{GeV}$ the bulk of the $m_{b b}$ distribution for the $t \bar{t}$ process is beyond the horizontal range of the plot.

(c) For Wh channel, $m_{\ell \ell}<80 \mathrm{GeV}$ or $m_{\ell \ell}>98 \mathrm{GeV}$.

(d) For $\mathrm{Zh}$ channel, $75 \mathrm{GeV}<m_{\ell \ell}<100 \mathrm{GeV}$.

(7) The scalar sum of transverse momenta of all reconstructed object: (e) For Wh channel:

$$
\begin{cases}H_{T} \leq 2500 \mathrm{GeV} & \sqrt{s}=3000 \mathrm{GeV} \\ H_{T} \leq 1100 \mathrm{GeV} & \sqrt{s}=1500 \mathrm{GeV}\end{cases}
$$

TABLE III. The cuts used for $W h$ channel and $Z h$ channel.

\begin{tabular}{|c|c|c|}
\hline Cuts & Wh-Cuts & $Z h$-Cuts \\
\hline Basic Cuts & \multicolumn{2}{|c|}{$\begin{array}{l}p_{T}^{\ell}>20 \mathrm{GeV}, N_{\ell}=2 \\
p_{T}^{j}>20 \mathrm{GeV}, N_{b}=2\end{array}$} \\
\hline & $N_{e} \geq 1$ & 1 OSSF Pair \\
\hline$m_{b b}$ & \multicolumn{2}{|c|}{$95 \mathrm{GeV} \leq m_{b b} \leq 130 \mathrm{GeV}$} \\
\hline$m_{\ell \ell}$ & $m_{\ell \ell} \leq 80 \mathrm{GeV}$ or $m_{\ell \ell} \geq 98 \mathrm{GeV}$ & $75 \mathrm{GeV} \leq m_{\ell \ell} \leq 100 \mathrm{GeV}$ \\
\hline$H_{T}$ & $\begin{cases}H_{T} \leq 2500 \mathrm{GeV} & \sqrt{s}=3000 \mathrm{GeV} \\
H_{T} \leq 1100 \mathrm{GeV} & \sqrt{s}=1500 \mathrm{GeV}\end{cases}$ & $\left\{\begin{array}{cc}H_{T} \leq 1500 \mathrm{GeV} & \sqrt{s}=3000 \mathrm{GeV} \\
H_{T} \leq 700 \mathrm{GeV} & \sqrt{s}=1500 \mathrm{GeV}\end{array}\right.$ \\
\hline
\end{tabular}


TABLE IV. The cross sections of all signal and background (BG) processes (with final states $b \bar{b} \ell^{+} \ell^{-}$) at $\sqrt{s}=1500,3000 \mathrm{GeV}$ for $P\left(e^{-}\right)=-0.8$. Note that for the VBF processes, $p_{T}^{\ell}>10 \mathrm{GeV}$ and $\left|\eta^{\ell}\right|<3.5$ are imposed at the generation level for the forward/backward charged lepton. We also quote the precision on the measurement of signal cross section that can be extracted with the given luminosity.

\begin{tabular}{|c|c|c|c|c|c|c|c|}
\hline \multirow[b]{2}{*}{$\sigma(\mathrm{fb})$} & & \multicolumn{3}{|c|}{$\sqrt{s}=3.0 \mathrm{TeV}, \mathcal{L}=4 \mathrm{ab}^{-1}$} & \multicolumn{3}{|c|}{$\sqrt{s}=1.5 \mathrm{TeV} \mathcal{L}=2 \mathrm{ab}^{-1}$} \\
\hline & & Before Cuts & $W h$-Cuts & Zh-Cuts & Before Cuts & $W h$-Cuts & Zh-Cuts \\
\hline \multirow[t]{2}{*}{ Signal } & $W h(\mathrm{VBF})$ & $1.97 \times 10^{0}$ & $7.26 \times 10^{-2}$ & $1.36 \times 10^{-3}$ & $9.62 \times 10^{-1}$ & $6.54 \times 10^{-2}$ & $2.37 \times 10^{-3}$ \\
\hline & $Z h(\mathrm{VBF})$ & $6.47 \times 10^{-1}$ & $3.49 \times 10^{-3}$ & $7.21 \times 10^{-2}$ & $2.03 \times 10^{-1}$ & $1.30 \times 10^{-3}$ & $2.87 \times 10^{-2}$ \\
\hline \multirow[t]{8}{*}{$\mathrm{BG}$} & $t t$ & $1.17 \times 10^{0}$ & $5.83 \times 10^{-4}$ & $6.10 \times 10^{-6}$ & $4.65 \times 10^{0}$ & $5.64 \times 10^{-3}$ & $8.05 \times 10^{-5}$ \\
\hline & $W Z(\mathrm{VBF})$ & $4.47 \times 10^{0}$ & $9.97 \times 10^{-3}$ & $2.16 \times 10^{-4}$ & $1.84 \times 10^{0}$ & $5.86 \times 10^{-3}$ & $1.96 \times 10^{-4}$ \\
\hline & $Z Z(\mathrm{VBF})$ & $1.92 \times 10^{0}$ & $4.21 \times 10^{-4}$ & $8.07 \times 10^{-3}$ & $5.92 \times 10^{-1}$ & $1.48 \times 10^{-4}$ & $2.88 \times 10^{-3}$ \\
\hline & $Z h$ & $5.88 \times 10^{-2}$ & $1.83 \times 10^{-4}$ & $4.15 \times 10^{-4}$ & $2.39 \times 10^{-1}$ & $4.10 \times 10^{-4}$ & $1.12 \times 10^{-3}$ \\
\hline & $Z W W$ & $4.01 \times 10^{-1}$ & $1.14 \times 10^{-3}$ & $4.97 \times 10^{-6}$ & $6.36 \times 10^{-1}$ & $2.02 \times 10^{-3}$ & $1.72 \times 10^{-5}$ \\
\hline & $Z Z Z$ & $5.06 \times 10^{-3}$ & $6.04 \times 10^{7}$ & $1.12 \times 10^{-5}$ & $9.79 \times 10^{-3}$ & $1.74 \times 10^{-6}$ & $2.34 \times 10^{-5}$ \\
\hline & Sum & $8.02 \times 10^{0}$ & $1.23 \times 10^{-2}$ & $8.72 \times 10^{-3}$ & $7.97 \times 10^{0}$ & $1.41 \times 10^{-2}$ & $4.32 \times 10^{-3}$ \\
\hline & & Precision $(\%)$ & 6.18 & 6.17 & Precision $(\%)$ & 9.53 & 13.5 \\
\hline
\end{tabular}

(f) For $Z h$ channel:

$$
\begin{cases}H_{T} \leq 1500 \mathrm{GeV} & \sqrt{s}=3000 \mathrm{GeV} \\ H_{T} \leq 700 \mathrm{GeV} & \sqrt{s}=1500 \mathrm{GeV}\end{cases}
$$

The cross sections for all processes after cuts are listed in Table IV for each channel, where in the last row, we also list the expected precision on the measurement of signal cross section with each channel assuming $\mathcal{L}=4000 \mathrm{fb}^{-1}$ for $\sqrt{s}=3000 \mathrm{GeV}$ and $\mathcal{L}=$ $2000 \mathrm{fb}^{-1}$ for $\sqrt{s}=1500 \mathrm{GeV}$.

The numbers listed in Tab. IV are for $\kappa_{W}=1$ and $\kappa_{Z}=1$. By assuming that the selection efficiency will not change significantly for different values, ${ }^{6}$ we can obtain the events at any other values of $\kappa_{W}$ and $\kappa_{Z}$ by

$$
\begin{aligned}
\mathcal{N}_{S}\left(\kappa_{W}, \kappa_{Z}\right) & =\mathcal{L} \times \sigma\left(\kappa_{W}, \kappa_{Z}\right) \\
& =\mathcal{L} \times \frac{\sigma^{\mathrm{obs}}}{\sigma_{0}\left(\kappa_{W}=1, \kappa_{Z}=1\right)} \times \sigma_{0}\left(\kappa_{W}, \kappa_{Z}\right),
\end{aligned}
$$

where $\sigma_{0}\left(\kappa_{W}, \kappa_{Z}\right)$ for $W h$ and $Z h$ at $\sqrt{s}=1500$ and $3000 \mathrm{GeV}$ can be constructed from the data listed in Table II as: $\sigma_{0}\left(\kappa_{W}, \kappa_{Z}\right)=\kappa_{W}^{2} \sigma_{W}+\kappa_{W} \kappa_{Z} \sigma_{W Z}+\kappa_{Z}^{2} \sigma_{Z}$. Then, assuming Poisson distribution for observed events, the negative log-likelihood (NLL) function is

\footnotetext{
${ }^{6}$ This is a reasonable assumption, as we did not use any selection cut that has direct dependence on the values of $\kappa_{W}$ and $\kappa_{Z}$.
}

$$
\begin{aligned}
\Delta \operatorname{NLL}\left(\kappa_{W}, \kappa_{Z}\right) & =\operatorname{NLL}\left(\kappa_{W}, \kappa_{Z}\right)-\mathrm{NLL}_{\min } \\
= & \sum_{i}\left(\left(\mathcal{N}_{B}^{i}+\mathcal{N}_{S}^{i}(1,1)\right) \log \left[\frac{\mathcal{N}_{B}^{i}+\mathcal{N}_{S}^{i}(1,1)}{\mathcal{N}_{B}^{i}+\mathcal{N}_{S}^{i}\left(\kappa_{W}, \kappa_{Z}\right)}\right]\right. \\
& \left.+\mathcal{N}_{S}^{i}\left(\kappa_{W}, \kappa_{Z}\right)-\mathcal{N}_{S}^{i}(1,1)\right),
\end{aligned}
$$

where the summation runs over all the channels we have considered. Then the bound with an $N-\sigma$ confidence interval corresponds to $\Delta \mathrm{NLL} \leq N^{2} / 2$.

Combining all these measurements, we can get the $68 \%$ $(\sim 1-\sigma)$ and $95 \%(\sim 2-\sigma)$ confidence level (C.L.) region. The results are shown in Fig. 5 in $\kappa_{W}-\kappa_{Z}$ (left panel), $\kappa_{W}-\lambda_{W Z}$ (middle panel) and $\kappa_{Z}-\lambda_{W Z}$ (right panel) plane respectively. The $68 \%$ region for each individual channel is also given in each plot which shows the complementarity among these channels. Note that although we did not optimize the cuts listed in Table III, they give a sensitivity very close to that achieved with a boosted decision tree using TMVA [45]. This is because with these cuts or any relatively similar ones, the signal to background ratio is very high.

Besides the C.L. region around SM point shown in Fig. 5, we can also estimate the luminosity that is needed to exclude some non-SM benchmark points. For this purpose, we will not combine $\sqrt{s}=1.5 \mathrm{TeV}$ and $3.0 \mathrm{TeV}$, as each has its own luminosity. The results are shown in Table V, and we see that significantly less data than the standard proposals is required to exclude these scenarios. In particular, the scenario with $\lambda_{W Z} \simeq-1$, which is very difficult to probe in other processes, can be probed with just a few inverse femtobarns. 

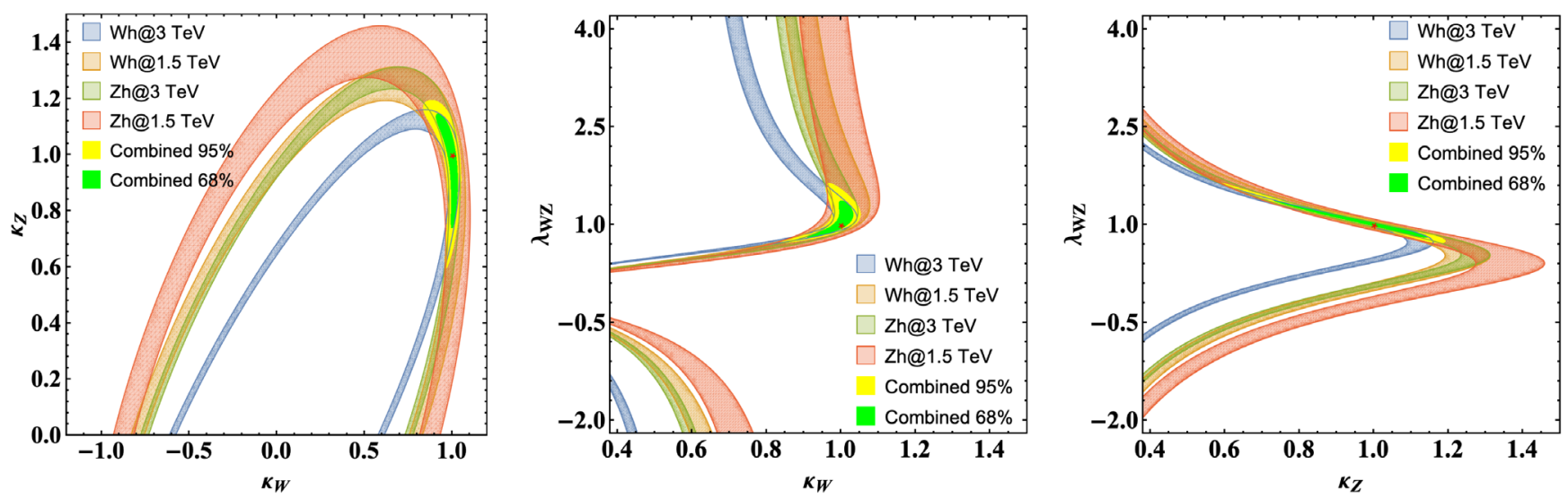

FIG. 5. The constraints in the $\kappa_{W}-\kappa_{Z}, \kappa_{W}-\lambda_{W Z}$, and $\kappa_{Z}-\lambda_{W Z}$ planes from the total rate measurements. We show the contours from the four different measurements at $68 \%$, and also show the combined constraints at $68 \%$ C.L. (95\% C.L.) in green (yellow). The SM values are indicated as red points.

\section{B. Differential distributions measurement}

Because of the growth with energy described in Sec. II, the dependence of the matrix element on $\hat{s}$ is different for $s$ - and $t / u$-channels. Therefore, the $s$ and $t / u$ channels depend on $\kappa_{W}$ and $\kappa_{Z}$ differently. Hence, the differential distribution shape will shift for different value of $\lambda_{W Z}$. This is illustrated in Fig. 6 in which we choose 3 different values of $\lambda_{W Z}$ with $\kappa_{W}=1$ and the distributions are all renormalized to 1 . Note that we have generated sufficiently large Monte Carlo samples to suppress the statistical fluctuations. Thus, the difference in the shape of the distributions for different values of $\lambda_{W Z}$ comes from the behavior of the matrix element.

In order to estimate the discrimination power, the MC events (including both signal and backgrounds) are used to obtain the "observed" $\left(\kappa_{W}=1\right.$ and $\left.\lambda_{W Z}=1\right)$ and "expected" (otherwise) distributions (shape) of $\sqrt{\hat{s}}$. The distributions as well as the total number of events are utilized to construct the extended likelihood by which we can determine the C.L. region for $\lambda_{W Z}$ using similar method in previous section. The $\triangle \mathrm{NLL}$ as a function of $\lambda_{W Z}$ when $\kappa_{W}=1$ is shown in Fig. 7. We find that utilizing the $\sqrt{\hat{s}}$ distribution can significantly improve the sensitivity.

From Fig. 7, we see that the sensitivity of the total cross section measurement is particularly weak for

TABLE V. The luminosity that is needed to exclude specific benchmark points at $95 \%$ C.L. against the SM case $\left(\kappa_{W}=1\right.$ and $\left.\kappa_{Z}=1\right)$.

\begin{tabular}{lcc}
\hline \hline Benchmark & $\sqrt{s}=3.0 \mathrm{TeV}$ & $\sqrt{s}=1.5 \mathrm{TeV}$ \\
\hline$\kappa_{W}= \pm 1, \kappa_{Z}=\mp 1$ & $3.4 \mathrm{fb}^{-1}$ & $14.1 \mathrm{fb}^{-1}$ \\
$\kappa_{W}=1, \kappa_{Z}=0$ & $29.3 \mathrm{fb}^{-1}$ & $243.3 \mathrm{fb}^{-1}$ \\
$\kappa_{W}=0, \kappa_{Z}=1$ & $62.1 \mathrm{fb}^{-1}$ & $1772.4 \mathrm{fb}^{-1}$ \\
\hline \hline
\end{tabular}

$1 \lesssim \lambda_{W Z} \lesssim 1.5$. This is because for fixed $\kappa_{W}$ as in Fig. 7, we have from Eq. (12)

$$
\frac{\partial \sigma}{\partial \lambda_{W Z}} \sim-\frac{\sigma_{W Z}}{\lambda_{W Z}^{2}}-\frac{2 \sigma_{Z}}{\lambda_{W Z}^{3}}
$$

Looking at Table II, we see that in all four scenarios we are interested in, this derivative vanishes for $\lambda_{W Z}$ between 1 and 1.5 , so the cross section in that region is changing very slowly and there is little sensitivity. On the other hand, the differential cross section in $\sqrt{\hat{s}}$ will change with fewer events near threshold and more events at higher energy as we move away from $\lambda_{W Z}=1$, allowing this analysis to break the approximate degeneracy in the total rate measurement.

\section{SUMMARY}

The nature of electroweak symmetry breaking and whether the Higgs has the precise properties given in the Standard Model is still not fully explored by data. The couplings of the Higgs to heavy gauge bosons are a particularly important probe of the mechanism that gives mass to the $W$ and $Z$. The ratio of those couplings, $\lambda_{W Z}$ (see Eqs. (2) and (5) for a precise definition) has been measured by the LHC to approximately $10 \%$ precision, but as yet we have essentially no information about the sign of $\lambda_{W Z}$.

Sign measurements are easiest in processes with treelevel interference; these processes will have very large deviations if the relative sign of a coupling is changed but the magnitude is kept constant. In this work we have studied $V V \rightarrow V h, V=W, Z$ using vector boson fusion at a high energy lepton collider. This process exhibits treelevel interference between diagrams proportional to the Higgs coupling to $W$ and that to $Z$. If there is a deviation from the SM prediction of $\lambda_{W Z}=1$, then this process 

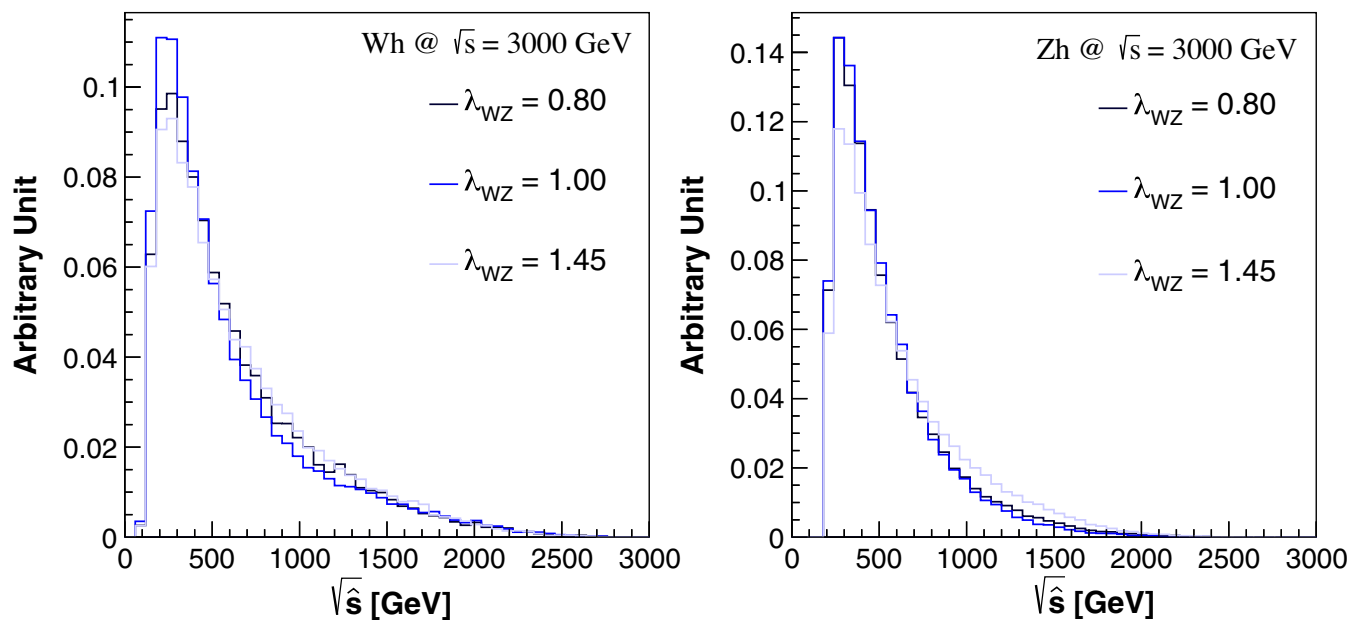

FIG. 6. The differential distributions of $\hat{s}$ for different values of $\lambda_{W Z}$ for $W h($ left) and $Z h$ (right) channel at $\sqrt{s}=3000 \mathrm{GeV}$.

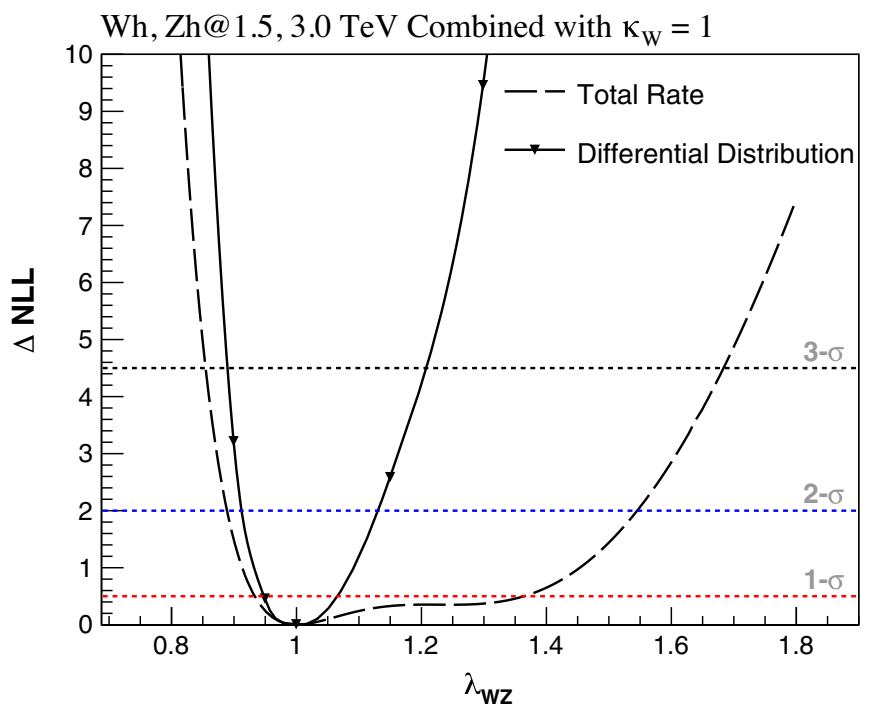

FIG. 7. The $\triangle$ NLL (see text for definition) for different values of $\lambda_{W Z}$ with $\kappa_{W}=1$ combining all $W h$ and $Z h$ channels at both 1.5 and 3.0 TeV CLIC. exhibits growth with energy. Therefore, at high energy there will be very large destructive interference between different processes as shown in Table II.

We have performed a study of this process at a potential future lepton collider with center of mass energies of 1.5 and $3 \mathrm{TeV}$. With simple cuts, one can get a signal to background ratio well above one. We have shown that combining the $e^{+} e^{-} \rightarrow \nu_{e} \bar{\nu}_{e} Z h$ and the $e^{+} e^{-} \rightarrow \nu_{e} e W h$ channels as well as combining measurements at different center of mass energies, one can measure the couplings of the gauge bosons to the Higgs with a reasonable precision. As discussed above, this channel is particularly powerful at probing $\lambda_{W Z} \simeq-1$, which can be excluded with a small fraction of the expected data. Finally, we have shown that including the $\sqrt{\hat{s}}$ differential distribution can further improve the measurement.

\section{ACKNOWLEDGMENTS}

We would like to thank Cheng-Wei Chiang for very useful discussions and communication. This work is supported in part by the Natural Sciences and Engineering Research Council of Canada (NSERC).
[1] C. H. Llewellyn Smith, High-energy behavior and gauge symmetry, Phys. Lett. 46B, 233 (1973).

[2] M. J. G. Veltman, Second threshold in weak interactions, Acta Phys. Pol. B 8, 475 (1977).

[3] B. W. Lee, C. Quigg, and H. B. Thacker, The Strength of Weak Interactions at Very High-Energies and the Higgs Boson Mass, Phys. Rev. Lett. 38, 883 (1977).
[4] B. W. Lee, C. Quigg, and H. B. Thacker, Weak interactions at very high-energies: The role of the Higgs boson mass, Phys. Rev. D 16, 1519 (1977).

[5] G. Passarino, Large masses, unitarity and one loop corrections, Phys. Lett. 156B, 231 (1985).

[6] G. Passarino, $W W$ scattering and perturbative unitarity, Nucl. Phys. B343, 31 (1990). 
[7] J. M. Cornwall, D. N. Levin, and G. Tiktopoulos, Derivation of gauge invariance from high-energy unitarity bounds on the s matrix, Phys. Rev. D 10, 1145 (1974); Erratum, Phys. Rev. D 11, 972 (1975).

[8] G. F. Giudice, C. Grojean, A. Pomarol, and R. Rattazzi, The strongly-interacting light Higgs, J. High Energy Phys. 06 (2007) 045.

[9] R. Contino, C. Grojean, M. Moretti, F. Piccinini, and R. Rattazzi, Strong double Higgs production at the LHC, J. High Energy Phys. 05 (2010) 089.

[10] G. Aad et al. (ATLAS Collaboration), Observation of a new particle in the search for the Standard Model Higgs boson with the ATLAS detector at the LHC, Phys. Lett. B 716, 1 (2012).

[11] S. Chatrchyan et al. (CMS Collaboration), Observation of a new boson at a mass of $125 \mathrm{GeV}$ with the CMS experiment at the LHC, Phys. Lett. B 716, 30 (2012).

[12] M. Szleper, The Higgs boson and the physics of $W W$ scattering before and after Higgs discovery, arXiv:1412 .8367 .

[13] B. Henning, D. Lombardo, M. Riembau, and F. Riva, Measuring Higgs Couplings without Higgs Bosons, Phys. Rev. Lett. 123, 181801 (2019).

[14] S. Dawson, The effective $W$ approximation, Nucl. Phys. B249, 42 (1985).

[15] Z. Kunszt and D. E. Soper, On the validity of the effective $W$ approximation, Nucl. Phys. B296, 253 (1988).

[16] P. Borel, R. Franceschini, R. Rattazzi, and A. Wulzer, Probing the scattering of equivalent electroweak bosons, J. High Energy Phys. 06 (2012) 122.

[17] A. M. Sirunyan et al. (CMS Collaboration), Measurement of vector boson scattering and constraints on anomalous quartic couplings from events with four leptons and two jets in proton-proton collisions at $\sqrt{s}=13 \mathrm{TeV}$, Phys. Lett. B 774, 682 (2017).

[18] A. M. Sirunyan et al. (CMS Collaboration), Observation of Electroweak Production of Same-Sign $W$ Boson Pairs in the Two Jet and Two Same-Sign Lepton Final State in ProtonProton Collisions at $\sqrt{s}=13 \mathrm{TeV}$, Phys. Rev. Lett. 120, 081801 (2018).

[19] M. Aaboud et al. (ATLAS Collaboration), Observation of electroweak $W^{ \pm} Z$ boson pair production in association with two jets in $p p$ collisions at $\sqrt{s}=13 \mathrm{TeV}$ with the ATLAS detector, Phys. Lett. B 793, 469 (2019).

[20] M. Aaboud et al. (ATLAS Collaboration), Observation of Electroweak Production of a Same-Sign $W$ Boson Pair in Association with Two Jets in $p p$ Collisions at $\sqrt{s}=$ $13 \mathrm{TeV}$ with the ATLAS Detector, Phys. Rev. Lett. 123, 161801 (2019).

[21] G. Aarons et al. (ILC Collaboration), International linear collider reference design report volume 2: Physics at the ILC, arXiv:0709.1893.

[22] H. Baer, T. Barklow, K. Fujii, Y. Gao, A. Hoang, S. Kanemura, J. List, H. E. Logan, A. Nomerotski, M. Perelstein et al., The international linear collider technical design report-volume 2: Physics, arXiv:1306.6352.

[23] M. Dong et al. (CEPC Study Group Collaboration), CEPC conceptual design report: Volume 2-Physics \& Detector, arXiv:1811.10545.
[24] M. Bicer et al. (TLEP Design Study Working Group Collaboration), First look at the physics case of TLEP, J. High Energy Phys. 01 (2014) 164.

[25] A. Abada et al. (FCC Collaboration), FCC-ee: The Lepton Collider, Eur. Phys. J. Special Topics 228, 261 (2019).

[26] L. Linssen, A. Miyamoto, M. Stanitzki, and H. Weerts, Physics and detectors at CLIC: CLIC conceptual design report, arXiv:1202.5940.

[27] P. Roloff, R. Franceschini, U. Schnoor, and A. Wulzer (CLIC, CLICdp Collaborations), The compact linear $\mathrm{e}^{+} \mathrm{e}^{-}$ collider (CLIC): Physics potential, arXiv:1812.07986.

[28] A. Costantini, F. De Lillo, F. Maltoni, L. Mantani, O. Mattelaer, R. Ruiz, and X. Zhao, Vector boson fusion at multi-TeV muon colliders, arXiv:2005.10289.

[29] C.-W. Chiang, X.-G. He, and G. Li, Measuring the ratio of $H W W$ and $H Z Z$ couplings through $\mathrm{W}^{+} \mathrm{W}^{-} \mathrm{H}$ production, J. High Energy Phys. 08 (2018) 126.

[30] Y. Chen, J. Lykken, M. Spiropulu, D. Stolarski, and R. Vega-Morales, Golden Probe of Electroweak Symmetry Breaking, Phys. Rev. Lett. 117, 241801 (2016).

[31] A. M. Sirunyan et al. (CMS Collaboration), Measurements of properties of the Higgs boson decaying into the fourlepton final state in pp collisions at $\sqrt{s}=13 \mathrm{TeV}$, J. High Energy Phys. 11 (2017) 047.

[32] M. Aaboud et al. (ATLAS Collaboration), Measurement of inclusive and differential cross sections in the $H \rightarrow Z Z^{*} \rightarrow$ $4 \ell$ decay channel in $p p$ collisions at $\sqrt{s}=13 \mathrm{TeV}$ with the ATLAS detector, J. High Energy Phys. 10 (2017) 132.

[33] A. M. Sirunyan et al. (CMS Collaboration), Measurements of properties of the Higgs boson decaying to a $W$ boson pair in pp collisions at $\sqrt{s}=13 \mathrm{TeV}$, Phys. Lett. B 791, 96 (2019).

[34] M. Aaboud et al. (ATLAS Collaboration), Measurements of gluon-gluon fusion and vector-boson fusion Higgs boson production cross-sections in the $H \rightarrow W W^{*} \rightarrow e \nu \mu \nu$ decay channel in $p p$ collisions at $\sqrt{s}=13 \mathrm{TeV}$ with the ATLAS detector, Phys. Lett. B 789, 508 (2019).

[35] G. Aad et al. (ATLAS, CMS Collaboration), Measurements of the Higgs boson production and decay rates and constraints on its couplings from a combined ATLAS and CMS analysis of the LHC pp collision data at $\sqrt{s}=7$ and $8 \mathrm{TeV}$, J. High Energy Phys. 08 (2016) 045.

[36] A. M. Sirunyan et al. (CMS Collaboration), Combined measurements of Higgs boson couplings in proton-proton collisions at $\sqrt{s}=13 \mathrm{TeV}$, Eur. Phys. J. C 79, 421 (2019).

[37] G. Aad et al. (ATLAS Collaboration), Combined measurements of Higgs boson production and decay using up to $80 \mathrm{fb}^{-1}$ of proton-proton collision data at $\sqrt{s}=13 \mathrm{TeV}$ collected with the ATLAS experiment, Phys. Rev. D 101, 012002 (2020).

[38] M. Cepeda et al., Report from working group 2: Higgs physics at the HL-LHC and HE-LHC, CERN Yellow Rep. Monogr. 7, 221 (2019).

[39] I. Low and J. Lykken, Revealing the electroweak properties of a new scalar resonance, J. High Energy Phys. 10 (2010) 053.

[40] H. Georgi and M. Machacek, Doubly charged Higgs bosons, Nucl. Phys. B262, 463 (1985).

[41] J. Alwall, R. Frederix, S. Frixione, V. Hirschi, F. Maltoni, O. Mattelaer, H. S. Shao, T. Stelzer, P. Torrielli, and M. Zaro, 
The automated computation of tree-level and next-toleading order differential cross sections, and their matching to parton shower simulations, J. High Energy Phys. 07 (2014) 079.

[42] T. Sjostrand, S. Mrenna, and P.Z. Skands, A brief introduction to PYTHIA 8.1, Comput. Phys. Commun. 178, 852 (2008).

[43] J. de Favereau, C. Delaere, P. Demin, A. Giammanco, V. Lemaître, A. Mertens, and M. Selvaggi (DELPHES 3
Collaboration), DELPHES 3, A modular framework for fast simulation of a generic collider experiment, J. High Energy Phys. 02 (2014) 057.

[44] E. Leogrande, P. Roloff, U. Schnoor, and M. Weber, A DELPHES card for the CLIC detector, arXiv:1909.12728.

[45] A. Hoecker, P. Speckmayer, J. Stelzer, J. Therhaag, E. von Toerne, and H. Voss, TMVA: Toolkit for multivariate data analysis, Proc. Sci., ACAT2007 (2007) 040 [arXiv:physics/ 0703039]. 